\title{
Diagnósticos de enfermagem para pacientes em risco de desenvolver úlcera por pressão
}

\author{
Nursing diagnoses for patients at risk of developing pressure ulcer \\ Diagnósticos de enfermería para pacientes en riesgo de desarrollar úlcera por presión
}

\author{
Thiago Moura de Araújo', Márcio Flávio Moura de Araújo', Joselany Áfio Caetano", \\ Marli Teresinha Gimenez Galvão", Marta Maria Coelho Damasceno" \\ ' Universidade Federal do Ceará. Faculdade de Farmácia, Odontologia e Enfermagem. \\ Programa de Pós-Graduação em Enfermagem. (Doutorando). Fortaleza-CE, Brasil. \\ " Universidade Federal do Ceará. Faculdade de Farmácia, Odontologia e Enfermagem. \\ Departamento de Enfermagem. Programa de Pós-Graduação em Enfermagem. Fortaleza-CE, Brasil.
}

Submissão: 14/3/2010 Aprovação: 21/2/2011

\section{RESUMO}

Estudo do tipo transversal, que objetivou identificar diagnósticos de enfermagem em pacientes críticos com risco para desenvolver úlcera por pressão. Foi realizado numa Unidade de Terapia Intensiva de Fortaleza-CE, com uma amostra intencional de treze sujeitos que apresentavam risco para úlcera por pressão, segundo a escala de Waterlow. Foi evidenciado um total de 45 diagnósticos de enfermagem, dos quais dezessete tinham significância clínica para o desenvolvimento de úlcera por pressão. Dentre estes, nove apresentaram frequência $\geq 80 \%$, destacando-se: Integridade da pele prejudicada (100\%), Deambulação prejudicada (87\%), Deglutição prejudicada ( $80 \%)$, Mobilidade no leito prejudicada $(80 \%)$ e Troca de gazes prejudicada ( $80 \%)$. O conhecimento destes diagnósticos de enfermagem é importante na detecção das repercussões e base clínica dessas lesões dermatológicas.

Descritores: Enfermagem; Úlcera por pressão; Diagnóstico de enfermagem; Unidade de terapia intensiva.

\section{ABSTRACT}

This cross-sectional study aimed to identify nursing diagnoses in critical patients at risk of developing pressure ulcer. It was carried out at an Intensive Care Unit in Fortaleza-CE, Brazil, using an intentional sample of thirteen subjects at risk of pressure ulcer, evaluated according to the Waterlow scale. In total, 45 nursing diagnoses were evidenced, seventeen of which were clinically significant for the development of pressure ulcer. The frequency levels for nine of these nursing diagnoses were $\geq$ $80 \%$, mainly: Impaired skin integrity $(100 \%)$, Impaired walking $(87 \%)$, Impaired swallowing ( $80 \%)$, Impaired bed mobility $(80 \%)$ and Impaired gas exchange (80\%). The knowledge of those nursing diagnoses is important in detecting the effects and clinical basis of skin lesions.

Key words: Nursing; Pressure ulcers; Nursing diagnosis; Intensive care unit.

\section{RESUMEN}

Estudio transversal, que objetivó identificar diagnósticos de enfermería en pacientes críticos con riesgo de desarrollar úlcera por presión. Fue llevado a cabo en una Unidad de Terapia Intensiva de Fortaleza-CE, usando una muestra intencional de trece sujetos con riesgo de úlcera por presión, según la escala de Waterlow. En total, fueron evidenciados 45 diagnósticos de enfermería, de los cuales diecisiete poseían significancia clínica para el desarrollo de úlcera por presión. Entre estos, nueve mostraron frecuencia $\geq$ $80 \%$, principalmente: Deterioro de la integridad cutanea (100\%), Deterioro de la deambulación (87\%), Deterioro de la deglución $(80 \%)$, Deterioro de la movilidad en la cama (80\%) y Deterioro del intercambio gaseoso (80\%). El conocimiento de los diagnósticos de enfermería es importante en la detección de los efectos clínicos y la base de las lesiones de la piel.

Palabras clave: Enfermería; Úlcera por presión; Diagnóstico de enfermería; Unidad de terapia intensiva. 


\section{INTRODUÇÃO}

As úlceras por pressão (UP) são complicações que poderão surgir em pacientes internados em Unidade de Terapia Intensiva (UTI), uma vez que estes estão expostos a inúmeros fatores de risco para tal ocorrência, como: instabilidade hemodinâmica; significativa limitação da mobilidade, decorrente de patologias diversas ou sequelas destas; estado geral comprometido; idade e condição nutricional ${ }^{(1,2)}$.

As UP são definidas como lesões cutâneas ou de partes moles, superficiais ou profundas, de etiologia isquêmica, secundária a um aumento de pressão externa, e localizam-se, usualmente, sobre uma proeminência óssea. O diagnóstico é feito por meio de métodos visuais que também classificam as úlceras em estágios, importantes na elaboração de estratégias terapêuticas. As UP são classificadas em níveis de I a IV de acordo com os danos observados nos tecidos, considerando as suas estruturas, podendo envolver a pele, tecidos subcutâneos, músculos, cartilagem e até mesmo ossos ${ }^{(3)}$.

Assim, a manutenção da integridade da pele em pacientes de UTI é um importante aspecto do cuidado de enfermagem, já que essas lesões constituem problemas graves e caros no cuidado prestado ao paciente. Por isso, para assegurar a qualidade da assistência, vários protocolos e instrumentos têm sido construídos. Dentre esses, a literatura apresenta escalas de avaliação que predizem a ocorrência de UP, sendo os mais conhecidos e utilizados os de Norton, Waterlow e Braden ${ }^{(4-7)}$.

A utilização de escalas de avaliação de risco para UP requer pouca habilidade do profissional enfermeiro, embora requeira mais tempo na assistência para sua avaliação e acompanhamento, mas é de extrema importância para o prognóstico, já que a identificação dos pacientes em risco permite a implementação de medidas preventivas precocemente, o que pode reduzir pela metade a incidência da UP $\mathrm{P}^{(4,8)}$. Porém, é importante entender que a avaliação de risco deve sempre estar acompanhada do julgamento clínico do enfermeiro, o qual nada mais é do que o processo de enfermagem. Entendido aqui, como uma atividade intelectual deliberada, por meio da qual a prática de enfermagem é abordada de uma maneira ordenada e sistemática ${ }^{(9)}$.

O processo de enfermagem é constituído de um conjunto de etapas inter-relacionadas: coleta de dados, diagnóstico de enfermagem, planejamento, implementação e avaliação, que focalizam a individualização do cuidado mediante uma abordagem de solução de problemas a qual se fundamenta em teorias e modelos conceituais de enfermagem ${ }^{(10)}$.

Dentre essas etapas, o diagnóstico de enfermagem tem merecido destaque por se tratar de uma etapa dinâmica, sistemática, organizada e complexa do processo de enfermagem, significando não apenas uma simples listagem de problemas, mas uma fase que envolve avaliação crítica e tomada de decisão.

A literatura é rica em publicações que destacam a importância do processo de enfermagem para a elevação da qualidade da assistência em saúde, com benefício tanto do paciente, através de um atendimento individualizado; como para a enfermagem. Ciente desta afirmação, e com base em experiência profissional, como enfermeiros intensivistas, e considerando a grande incidência de UP em UTI. Este estudo teve como objetivo identificar os diagnósticos de enfermagem mais presentes em pacientes com risco para UP em uma UTI de Fortaleza-CE, Brasil.

\section{REFERENCIAL TEÓRICO}

Na escolha de um método de avaliação de risco para o desenvolvimento de UP, é importante considerar a eficácia e a facilidade de aplicação do instrumento de medida, embora que vários métodos de avaliação de risco para UP têm sido desenvolvidos, através da análise e pontuação dos fatores de risco predisponentes ao desenvolvimento da úlcera, na sua grande maioria são opiniões de especialistas ou adaptações de instrumentos já existentes ${ }^{(2)}$.

A European Pressure Ulcer Advisory Panel (EPUAP), órgão que se originou em Londres, em 1996, que, periodicamente, se reúne e apresenta diretrizes de prevenção e tratamento baseadas em evidências, recomenda que um instrumento de avaliação de risco deve incluir: condição geral e avaliação da pele, mobilidade, umidade, incontinência, nutrição e dor ${ }^{(7)}$. As escalas de Norton, Braden e Waterlow estão entre as mais utilizadas no mundo, embora a única que apresenta a avaliação da pele como fator de risco seja a escala de Waterlow ${ }^{(8)}$.

A escala de Waterlow foi baseada na de Norton, porém com avaliação de um maior número de itens que foram obtidos após longa investigação dos fatores etiológicos das UP ${ }^{(9)}$. Desenvolvimento de modelos de avaliação de risco para pacientes hospitalizados representa uma tarefa complexa, cujos critérios a serem incluídos neste devem atender o objetivo a ser alcançado, a exemplo temos que escalas de avaliação de risco para UP buscam diagnosticar a presença de lesões, assim como sua evolução, de maneira a identificar o paciente com maior risco de predisposição.

Para ser realizado um cuidado de enfermagem adequado às exigências de um cliente em estado crítico é preciso uma estrutura organizacional específica, tanto em relação aos recursos humanos, quanto aos recursos físicos e materiais, inclusive com criação de instrumentos de coleta de dados que permita a identificação das necessidades humanas básicas do paciente e que também estabeleça uma comunicação multiprofissional, garantindo a continuidade da assistência de enfermagem.

O referencial teórico que norteou este trabalho foi fundamentado no modelo conceitual de Wanda Horta, que se baseia nas necessidades humanas básicas propostas por Maslow e na classificação de Mohana. Optamos pelo modelo de Horta por considerá-lo um modelo que permite a avaliação do paciente como um todo indivisível, com seus componentes bio-psico-sócio-espirituais, o qual contém as seguintes fases: a) histórico de enfermagem, b) diagnóstico de enfermagem, c) plano assistencial, d) prescrição de enfermagem, e) evolução de enfermagem e f) prognóstico de enfermagem ${ }^{(4,11)}$.

\section{MÉTODO}

Trata-se de uma pesquisa descritiva do tipo transversal. O estudo foi conduzido num hospital do município de 
Fortaleza-CE de atenção terciária na área de urgência e emergência durante o período de fevereiro a abril de 2009.

A amostragem foi intencional e alguns critérios de inclusão foram estabelecidos, a saber: ter idade $\geq 18$ anos; apresentar familiar ou responsável nos casos de pacientes inconscientes; estar internado a mais de 24 horas na UTI; apresentar risco para UP segundo a escala de avaliação de risco de Waterlow; não ser acometido por doença rara ou quadro clínico interrogado. Durante a execução da investigação um total de 21 pacientes foram internados nesta UTI. Contudo, apenas treze contemplaram os critérios de inclusão supracitados.

A coleta de dados ocorreu nos meses de fevereiro a março de 2009. Ela foi dividida em duas etapas. Primeiramente, foi realizada a avaliação de risco para UP, a partir da escala de Waterlow, adaptada e validada para língua portuguesa, que consistiu na avaliação de 10 itens: índice de massa corporal (IMC); tipo de pele, sexo/idade, continência, mobilidade, subnutrição do tecido celular, deficiência neurológica, cirurgia de grande porte/trauma, apetite, sonda nasogástrica (SNG) ou sonda nasoenteral (SNE) e medicação. Cada item possui uma categorização própria, com valores que variam entre 0 e 5 . Na avaliação dos pacientes, escores 10-14 pontos, 15-19 pontos e $\geq 20$ pontos são preditores de risco, alto risco e altíssimo risco de desenvolvimento de UP, respectivamente ${ }^{(12,13)}$. Vale pontuar que, além do exame físico, a consulta ao prontuário e aos familiares também ocorreu no levantamento de alguns destes dados.

$\mathrm{Na}$ segunda etapa foi aplicada a sistematização da assistência de enfermagem com a realização do histórico de enfermagem e exame físico para levantamento dos diagnósticos de enfermagem nos pacientes com risco para UP.

Para nortear essa etapa foi adotado um instrumento, já validado e publicado, de sistematização de enfermagem específico para unidades de cuidados intensivos. Os autores do referido material, ao elaborar este instrumento utilizaram como referencial teórico a Teoria das Necessidades Humanas de Wanda Horta. Na validação desse material, os juízes concordaram que este seria suficiente para o estabelecimento de diagnósticos de enfermagem, favorecendo o desenvolvimento da sistematização da assistência de enfermagem ao paciente crítico, em centros de terapia intensiva ${ }^{(14)}$.

Para a investigação das respostas humanas foi averiguada a presença ou ausência dos fatores relacionados/risco de cada diagnóstico de enfermagem presentes na versão brasileira 2007-2008 da classificação de diagnósticos de enfermagem da NANDA (North American Nursing Diagnosis Association) (15). Para auxiliar na análise dos diagnósticos de enfermagem, foi utilizado o seguinte raciocínio crítico: análise de agrupamentos de indicadores, criação de uma lista de problemas suspeitados, descarte de diagnósticos similares, escolha dos rótulos diagnósticos mais específicos e declaração dos problemas e suas causas ${ }^{(16,17)}$.

Os preceitos éticos e legais a serem seguidos nas investigações envolvendo seres humanos foram respeitados, conforme preconiza a Resolução 196/96, do Conselho Nacional de Saúde. Para tanto, o projeto em pauta foi apreciado pelo Comitê de Ética em Pesquisa da instituição, tendo sua aprovação no protocolo de no. $86145 / 08$, e obtido o consentimento livre e informado dos participantes após o esclarecimento sobre os objetivos e a forma de condução da pesquisa. Para os pacientes sem condições de fornecer o consentimento esse foi obtido junto a familiares ou responsáveis.

\section{RESULTADOS}

\section{Avaliação de risco para UP}

A faixa etária dos sujeitos foi estratificada da seguinte maneira: 50 a 64 anos; 65 a 74 anos; 75 a 80 anos e acima de 80 anos. Nas três primeiras categorias houve dois participantes em cada, enquanto na última houve sete participantes.

Quanto ao tipo de pele foi observada em oito pacientes a presença de pele muito fina e com edema. Onze pacientes estavam com IMC acima da média. Foi observado que oito pacientes tinham incontinência ocasional e três, dupla incontinência. Em relação ao fator de risco mobilidade, todos pacientes estavam com mobilidade restrita/contido. Entre os pacientes críticos avaliados, a insuficiência cardíaca e a doença vascular periférica ocorriam em quatro e dois sujeitos, respectivamente. Nenhum dos sujeitos era tabagista e apenas um apresentava anemia.

A deficiência neurológica foi observada em oito sujeitos, sendo quatro relacionada exclusivamente ao Diabetes Mellitus e as outras com a paraplegia motora/sensitiva. Entre os quatro pacientes com paraplegia motora, dois também eram diabéticos. O fator de risco cirurgia/trauma só foi identificado em dois pacientes. No quesito apetite, predominou (sete sujeitos) o item "somente líquido". Uma paciente apresentou o uso de SNG/E devido a caquexia. O fator de risco medicação, referente a altas dosagens de esteróides, citotóxicos e antiinflamatórios, não pontuou nesse grupo de pacientes.

Sete pacientes apresentaram alto risco e seis, altíssimo risco de desenvolver UP, segundo o Método de Waterlow. Dentre os sujeitos com alto risco para desenvolvimento de UP, o fator predominante foi o sexo (11 mulheres). Enquanto nos sujeitos com altíssimo risco para desenvolver UP, os fatores predominantes foram: deficiência neurológica (8); idade acima de 80 anos (6); subnutrição do tecido celular (12); apetite (6); ocasional continência (5) e cirurgia/trauma (2).

\section{Diagnósticos de Enfermagem}

Na tabela 1 estão apresentados os diagnósticos de enfermagem identificados nos treze pacientes com risco de UP, segundo a Taxonomia II da NANDA. Um total de 45 diagnósticos de enfermagem foram encontrados, sendo que 17 tinham significância clínica para o desenvolvimento de lesões dermatológicas como as UP. Dentre estes dezessete diagnósticos, nove apresentaram freqüência $\geq 80 \%$, com ênfase para os diagnósticos: Integridade da pele prejudicada (100\%), Deambulação prejudicada (87\%), Deglutição prejudicada (80\%), Mobilidade no leito prejudicada (80\%) e Troca de gazes prejudicada (80\%).

O diagnóstico de enfermagem Integridade da pele prejudicada esteve presente em todos os sujeitos. Seis diagnósticos de enfermagem foram encontrados em mais de um fator de risco do Método de Waterlow, a saber: Déficit no autocuidado para banho/higiene, Deambulação prejudicada, Risco de glicemia 
Tabela 1. Distribuição dos diagnósticos de enfermagem em pacientes de uma UTI em relação à Escala de Waterlow. Fortaleza-CE, 2009.

\begin{tabular}{lcc}
\hline Diagnósticos de Enfermagem & Fatores de risco da Escala de Waterlow & $\boldsymbol{f}(\boldsymbol{n}=\mathbf{1 3})$ \\
\hline Déficit no autocuidado para banho/higiene & Mobilidade e idade & 13 \\
Constipação & Continência & 6 \\
Deambulação prejudicada & Mobilidade e idade & 13 \\
Debito cardíaco diminuído & Subnutrição do tecido celular & 5 \\
Deglutição prejudicada & Apetite & 12 \\
Diarréia & Continência & 4 \\
Eliminação urinária prejudicada & Continência & 9 \\
Risco de glicemia instável & Continência & 4 \\
Incontinência urinária & Deficiência neurológica e Apetite & 2 \\
Integridade da pele prejudicada & Mobilidade & 13 \\
Mobilidade física prejudicada & Mobilidade e Deficiência neurológica & 10 \\
Mobilidade no leito prejudicada & Subnutrição do tecido celular & 12 \\
Risco de disfunção neurovascular periférica & Cipo de pele e Subnutrição do tecido celular & 5 \\
Perfusão tissular ineficaz & Subnutrição do tecido celular & 9 \\
Recuperação cirúrgica retardada & Subnutrição do tecido celular & 12 \\
Troca de gazes prejudicada & & 7 \\
Resposta disfuncional ao desmame ventilatório & & 3 \\
\hline
\end{tabular}

prejudicada, Integridade da pele prejudicada e Troca de gases prejudicada apresentaram freqüência em mais da metade dos participantes com altíssimo risco para UP. Por sua vez, naqueles com alto risco isto só foi observado no diagnóstico Mobilidade no leito prejudicada (Tabela 2).

\section{DISCUSSÃO}

A escala de Waterlow, traduzida para língua portuguesa pouca mais de cinco anos, vem mostrando-se como um instrumento adequado com especificidade e sensibilidade para predizer risco para UP(6).

Neste estudo, a pontuação dos pacientes variou de 15 a

instável, Integridade da pele prejudicada, Mobilidade física prejudicada e Perfusão tissular ineficaz (Tabela 1).

O diagnóstico de enfermagem Integridade da pele prejudicada, por exemplo, apresentou associação com os seguintes fatores de risco da Escala de Waterlow: idade, tipo de pele e subnutrição do tecido celular. Em contrapartida, o fator de risco idade possibilitou a formulação dos seguintes diagnósticos de enfermagem: Déficit no autocuidado para banho/ higiene, Deambulação prejudicada e Integridade da pele prejudicada (Tabela 1).

Os diagnósticos de enfermagem Déficit no autocuidado para banho/ higiene, Deambulação prejudicada, Deglutição

\section{Tabela 2. Distribuição dos diagnósticos de enfermagem segundo o risco para UP numa UTI. Fortaleza-CE, 2009.}

\begin{tabular}{|c|c|c|c|}
\hline \multirow{2}{*}{ Diagnósticos de Enfermagem } & \multicolumn{3}{|c|}{$\begin{array}{c}\text { Classificação de risco para úlcera } \\
\text { por pressão }\end{array}$} \\
\hline & Alto Risco & Altíssimo Risco & $f$ \\
\hline Déficit no autocuidado para banho/higiene & 3 & 10 & 13 \\
\hline Constipação & 2 & 4 & 6 \\
\hline Deambulação prejudicada & 2 & 11 & 13 \\
\hline Debito cardíaco diminuído & 2 & 3 & 5 \\
\hline Deglutição prejudicada & 4 & 8 & 12 \\
\hline Diarréia & 2 & 2 & 4 \\
\hline Eliminação urinária prejudicada & 4 & 5 & 9 \\
\hline Risco de glicemia instável & 1 & 3 & 4 \\
\hline Incontinência urinária & - & 2 & 2 \\
\hline Integridade da pele prejudicada & 4 & 9 & 13 \\
\hline Mobilidade física prejudicada & 5 & 5 & 10 \\
\hline Mobilidade no leito prejudicada & 7 & 5 & 12 \\
\hline Risco de disfunção neurovascular periférica & 2 & 3 & 5 \\
\hline Perfusão tissular ineficaz & 3 & 6 & 9 \\
\hline Recuperação cirúrgica retardada & 1 & 2 & 3 \\
\hline Troca de gazes prejudicada & 5 & 7 & 12 \\
\hline Resposta disfuncional ao desmame ventilatório & 4 & 3 & 7 \\
\hline
\end{tabular}

42 pontos, estabelecendo uma média de 24,6 pontos. Assim, com pontuação superior a 20 pontos, os pacientes enquadram-se no grupo de altíssimo risco. Em pesquisas anteriores, fica evidente também o risco de desenvolvimento de UP em pacientes críticos, embora a avaliação e medidas de prevenção não sejam destacadas na mesma proporção ${ }^{(18,19)}$. A avaliação de risco para UP em pacientes de UTI tem sido uma prática ainda não muito comum, mas que já vêem apresentando resultados em estudos na área da enfermagem. Embora tenhamos utilizado a escala de Waterlow, outras escalas de avaliação de risco para UP vêem sendo utilizada como a de Braden, Norton e Gosnell. Essas escalas também apresentam fácil utilização na prática clínica e evidências de confiabilidade e sensibilidade em estudos da área ${ }^{(18,19)}$

As UTI são pontuadas como local propício para desenvolver uma UP por diminuir a mobilidade dos pacientes associado ao agravamento da terapêutica dos mesmos. O confinamento no leito e o número excessivo de dispositivos e tecnologias duras dificultam a mobilidade e manobras de prevenção para UP como a mudança de decúbito.

Por exemplo, dentro dos resultados referentes à escala, podemos identificar o IMC acima da média em 11 pacientes do estudo. O peso elevado dos pacientes dificulta a mobilidade no leito e as manobras de mudança de decúbito. Quando o excesso de peso está associado ao Diabetes, o risco para formação de uma UP é aumentado devido ao déficit neurológico periférico e a diminuição vascularização venosa.

A escala de Waterlow apresenta um item importante e diferencial. O tipo de pele do paciente pode ser avaliado e gerar risco para 
UP. Tal fato pode ser confirmado quando observamos que a maioria dos pacientes pontuou nos item do tipo de pele e encontra-se em alto ou altíssimo risco. A avaliação da pele pode ser observada por toda equipe de saúde e sinaliza alterações no quadro clínico do paciente. O edema pode ser evidenciado e está associado a patologias específica como também a diminuição da mobilidade.

A Sistematização da Assistência de Enfermagem (SAE) pode levantar questionamentos para o risco e elaboração de planos de cuidados para pacientes em UTI. Os diagnósticos de enfermagem encontrados nos pacientes em risco para UP, em sua maioria, tem ligação direta com os fatores de risco da escala de Waterlow. Por exemplo, os diagnósticos de enfermagem: déficit do autocuidado para banho/ higiene, deambulação prejudicada, mobilidade no leito prejudicada estão relacionados com os itens de mobilidade e déficit neurológico (paraplegia e diabetes).

As características definidoras ajudaram a identificar os diagnósticos de enfermagem e relacionar aos fatores de risco da escala de Waterlow. Dentro dos diagnósticos, a integridade da pele prejudicada foi o que apresentou maior prevalência. A classificação do tipo de pele em saudável, muito fina, com edema, quebradiça, úmida e descorada ajuda o enfermeiro a ter maior precisão na hora de pontuar na escala e detectar este diagnóstico. A referida resposta humana, também apresenta fatores relacionados externos que podem ser logo contemplados em outros itens da escala como: extremos de idade, imobilização física e medicamentos. Além dos fatores externos podemos observar os fatores internos, nesse caso, especialmente as proeminências ósseas e fatores nutricionais apresentam relação direta com risco e evolução das UP(20)

A deglutição prejudicada está relacionada ao apetite e ao uso de SNG/E. Ela apresenta ainda características definidoras comuns ao pacientes em UTI, como infecções pulmonares recorrentes, regurgitação de conteúdo gástrico e limitação do volume de alimento. Dentro dos fatores relacionados podemos enfatizar a obstrução mecânica, decorrente da entubação orotraqueal, comum nesses sujeitos.

Por sua vez, o déficit na deglutição pode gerar comprometimento nutricional, embora publicações identifiquem a dieta enteral como adequada e eficiente para repor as necessidades calóricas do indivíduo. O uso de dispositivos para alimentação agrega prejuízos na fala, podendo o enfermeiro associar esse diagnóstico ao de comunicação verbal prejudicada. A comunicação verbal prejudicada dificulta o levantamento de dados no processo de enfermagem, sendo a resposta humana aos estímulos do paciente uma ferramenta para construção do plano de ação ${ }^{(21,22)}$.

\section{CONSIDERAÇÕES FINAIS}

Apesar de ter tido limitações, como ter sido desenvolvida com um número limitado de sujeitos e referentes apenas uma UTI, a pesquisa pôde constatar a viabilidade clínica da escala de Waterlow. Além disso, a investigação dos fatores de risco da escala auxiliou na contemplação de muitos diagnósticos de enfermagem, favorecendo o conhecimento precoce de ações de prevenção para UP. O item tipo de pele da escala e o diagnóstico Integridade da pele prejudicada estiveram relacionados. Assim, as medidas de prevenção puderam ser direcionadas de acordo com cada tipo de pele e o risco identificado.

Podemos observar que o risco de desenvolver UP inerente às UTI pode ser percebido e prevenido com auxilio da SAE e protocolos específicos de prevenção de úlcera. A proposta da pesquisa em realizar um estudo de caso de uma UTI específica pode gerar discussões para formação de novos protocolos, contemplando a necessidade de cada instituição. A associação da escala de Waterlow com a SAE pode nos atentar para diagnósticos que estão relacionados indiretamente com o risco para formação de UP como deglutição prejudicada, diarréia, risco para glicemia instável e risco para disfunção neurovascular periférica, entre, repercutindo numa melhor assistência de enfermagem.

\section{REFERÊNCIAS}

1. Rabeh SAN, Caliri MHL. Prevenção e tratamento de úlceras de pressão: práticas de graduados de enfermagem. Rev paul. enferm. 2002; 21(2): 133-9.

2. Fernandes NCS, Torres C, Vieira D. Fatores de risco e condições predisponentes pra úlcera de pressão em pacientes de terapia intensiva. Rev. Eletr. Enf. [Internet] 2008 [citado 28 set 2009]; 10(3):733-46. Disponível em: http.www. fen.ufg.br/revistalv10 \n3lv10n3a19.htm.

3. Blanes L, Duarte IS, Calil JF, Ferreira LM. Avaliação clínica e epidemiológica das úlceras de pressão em pacientes internados no Hospital São Paulo. Rev Ass Med Bras 2004;50(2): 182-7

4. Gould, D, Goldstone L, Kelly D, Gammon J. Examining the validity of pressure ulcer risk assessment scales: a replication study. Int J Nurs Stud 2004;41(3): 331-9.
5. Rangel, EML. Conhecimento, práticas e fontes de informação de enfermeiros de um hospital sobre a prevenção e tratamento da úlcera de pressão [dissertação]. Ribeirão Preto (SP): Universidade de São Paulo, 2004. Mestrado em Enfermagem.

6. Rocha, ABL; Barros, SMO. Avaliação de risco de úlcera por pressão: propriedades de medida da versão em português da escala de Waterlow. Acta paul. enferm. 2007;20(2):143-50 .

7. Maklebust J, Sieggreen M. Pressure ulcer: guidelines for prevention and nursing management. Pennsylvania: Spring Corpor, 1996.

8. Dealey C. Cuidando de Feridas um guia para as enfermeiras. Tradução: Eliane Kanner. $2^{\text {a }}$ edição. São Paulo: Atheneu; 2001. 
9. Araújo TM. Acurácia de escalas para avaliação de risco para úlcera por pressão em pacientes críticos [dissertação]. Fortaleza (CE): Universidade Federal do Ceará; 2009. Mestrado em Enfermagem.

10. Galdeano LE, Rossi LA, Nobre LF, Ignácio DS. Diagnósticos de enfermagem de pacientes no período transoperatório de cirurgia cardíaca. Rev. Latino-Am. Enfermagem 2003;11(2): 199-206.

11. Figueiredo RM, Zem-Mascarenhas $\mathrm{SH}$, Napoleão AA, Camargo AB. Caracterização da produção do conhecimento sobre sistematização da assistência de enfermagem no Brasil. Rev Esc Enferm USP, 2006;40(2): 299-303.

12. Fernandes, LM. Úlcera de pressão em pacientes críticos hospitalizados: uma revisão integrativa da literatura [dissertação]. São Paulo (SP): Universidade de São Paulo; 2000. Mestrado em Enfermagem.

13. Waterlow, J. A policy that protects: the Waterlow pressure sore prevention/treatment policy. In: Horne, EM; Cowan, T. Staff nurse's survival guide. 2.ed. London: Mosby; 1992.

14. Bittar DB, Pereira LV, Lemos RCA. Sistematização da assistência de enfermagem ao paciente crítico: proposta de instrumento de coleta de dados. Texto Contexto Enferm 2006;15(4): 617-28.

15. North American Nursing Diagnosis Association. Diagnósticos de enfermagem da NANDA: definições e classificação (2007-2008). Porto Alegre: Artmed; 2008.
16. Lefevre RA. Aplicação do processo de enfermagem-promoção do cuidado colaborativo. 5.Ed. Porto Alegre: Artmed. 2005.

17. Risner PB. Diagnosis: analysis and synthesis of data. In: Cristensen PJ, Kenney JW. Nursing process: application of conceptual models. St. Louis: Mosby; 1990. p. 132-57.

18. Fernandes LM, Caliri MHL. Uso da escala de Braden e de Glasgow para identificação de risco para úlcera por pressão em pacientes internados em centro de terapia intensiva. Rev. Latino-Am. Enfermagem 2008;16(6):947-52.

19. Barros SKSA, Anami EHT, Elias ACGP, Hashimoto MLY, Tsuda MS, Dorta PO, et al. Aplicação de protocolo para prevenção de úlcera por pressão em unidade de terapia intensiva. Semina: Ciências Biológicas e da Saúde 2002;23(1):25-32.

20. Gonçalves LA, Garcia PC, Toffoleto MC, Telles SCR, Padilha KG. Necessidades de cuidados de enfermagem em terapia intensiva: evolução diária dos pacientes segundo o Nursing Activities Score (NAS). Rev Bras Enferm 2006; 9(1):56-60

21. Ciampone JT, Gonçalves LA, Maia FOM, Padilha KG. Necessidade de cuidados de enfermagem e intervenções terapêuticas em UTI: estudo comparativo entre pacientes idosos e não idosos. Acta paul. enferm. 2006;19(1 Suppl 1):28-35.

22. Fernandes LM; Caliri MHL; Haas VJ. Efeitos de intervenções educativas no conhecimento dos profissionais de enfermagem sobre prevenção de úlceras pressão. Acta paul. enferm. 2008;21(2):305-11. 